\title{
Optimal Control of Open Quantum Systems: Cooperative Effects of Driving and Dissipation
}

\author{
R. Schmidt, ${ }^{1}$ A. Negretti, ${ }^{2}$ J. Ankerhold, ${ }^{1}$ T. Calarco,${ }^{2}$ and J. T. Stockburger ${ }^{1}$ \\ ${ }^{1}$ Institut für Theoretische Physik, Universität Ulm, Albert-Einstein-Allee 11, 89069 Ulm, Germany. \\ ${ }^{2}$ Institut für Quanteninformationsverarbeitung, Universität Ulm, Albert-Einstein-Allee 11, 89069 Ulm, Germany.
}

(Dated: August 22, 2011)

\begin{abstract}
We investigate the optimal control of open quantum systems, in particular, the mutual influence of driving and dissipation. A stochastic approach to open-system control is developed, using a generalized version of Krotov's iterative algorithm, with no need for Markovian or rotating-wave approximations. The application to a harmonic degree of freedom reveals cooperative effects of driving and dissipation that a standard Markovian treatment cannot capture. Remarkably, control can modify the open-system dynamics to the point where the entropy change turns negative, thus achieving cooling of translational motion without any reliance on internal degrees of freedom.
\end{abstract}

PACS numbers: 03.65.Yz, 02.30.Yy, 03.67.-a, 05.70.Ln

Introduction. The control of quantum dynamics for the accurate preparation of a prescribed quantum state by a tailored time-dependent field is a task of key importance in quantum physics and related disciplines. With increasing complexity of devices for quantum information processing the destructive role of environmental fluctuations has become a severe limitation to further progress. For example, neutral atoms or ions in electromagnetic traps are exposed to fluctuations of (comparatively hot) chip surfaces [1, while in superconducting circuits diffusing charges and electromagnetic fluctuations affect fidelities quite substantially [2].

In this context, optimal control theory (OCT) has emerged as a key ingredient in strategies to tame the effect of decoherence and other imperfections either directly by mitigating their effect or indirectly by speeding up operations. In limiting cases or under idealized conditions OCT has already been used to increase the fidelity of simple quantum gates [3 7] or to construct more complex protocols [8]. Generally, however, OCT has treated environmental interactions mostly by heuristic or approximate methods so far. A simple strategy has been followed in Ref. 9], where the impact of a heat bath was taken into account by assuming an initial thermal state while neglecting its effect completely during its dynamics. Some progress has been achieved within fully dynamical approaches based on standard Markovian master equations. Unfortunately, these schemes have severe drawbacks: First, they become inconsistent for strong control fields unless additional field-dependent memory terms in the dissipator are introduced [10, 11]. Second, the rotating wave approximation (RWA), which is usually performed on the system-environment interaction in order to obtain a master equation with complete positivity, is known to be unreliable in driven systems [10. Third, at sufficiently low temperatures or for reservoirs with structured spectral mode densities the true dynamics of open quantum systems is non-Markovian. These errors in the dynamics may be further amplified by OCT search algo- rithms. As a result, OCT computations using standard master equations yield mismatched fields, which perform poorly when applied to a realistic setting.

In this Letter we present a treatment of optimal control of open quantum systems which is not susceptible to these problems. Stochastic Liouville-von Neumann (SLN) equations [12, 13, provide an approach to quantum dissipation in a driven system which is both conceptually transparent and formally exact. Field-induced modifications of environmental effects are thus included a priori. From the SLN equations one arrives naturally at the definition of a state-costate pair [14] of dynamical variables with the simple first-order equations of motion required by gradient-based and related OCT methods.

We outline the salient features of our OCT technique (for details see Supplemental Material [15]) and apply it to a simple model. Notably, it turns out that control fields extracted from a RWA-based master equation differ substantially from exact ones obtained within the SLN scheme. This leads even to the counterintuitive phenomenon of control-induced cooling, which is completely missing in the RWA approach.

Open-system dynamics and control algorithm.-We start with the exact stochastic equation of motion 12

$$
\begin{aligned}
\varrho_{\xi, \nu}(t)= & -\frac{i}{\hbar}\left[H_{\mathrm{s}}, \varrho_{\xi, \nu}(t)\right]-\frac{i}{\hbar}\left[H_{\mathrm{c}}, \varrho_{\xi, \nu}(t)\right] \\
& +\frac{i}{\hbar} \xi(t)\left[q, \varrho_{\xi, \nu}(t)\right]+\frac{i}{2} \nu(t)\left\{q, \varrho_{\xi, \nu}\right\}
\end{aligned}
$$

for noisy samples $\varrho_{\xi, \nu}(t)$ of the density matrix of an open quantum system. The system is characterized by a Hamiltonian $H_{\mathrm{s}}$ governing its autonomous motion, augmented by a term $H_{\mathrm{c}}(t)$ describing the influence of timedependent control fields $u_{j}(t)$. It interacts bilinearly with a dissipative environment whose quantum statistical force-force correlation function is mapped to the noise statistics as follows [12]: (i) the autocorrelation of $\xi$ matches the quantum noise of the reservoir, (ii) the crosscorrelations of $\xi$ and $\nu$ match the dynamical response 
of the environment and (iii) the correlations $\left\langle\nu(t) \nu\left(t^{\prime}\right)\right\rangle$ vanish identically. According to the last condition $\nu(t)$ is a complex variable with random phase. The nonvanishing correlations are identical to the real and imaginary parts of the kernel defining the Feynman-Vernon influence functional [16, 17, from which Eq. (1) can be derived without approximations.

The fluctuations and the dynamical response of the thermal environment are fully characterized by its temperature and spectral density $J(\omega)$, which in the present case will be taken as Ohmic (proportional to $\omega$ up to a UV cutoff $\left.\omega_{c}\right)$. Both $1 / \omega_{c}$ and, more important, the thermal time $\hbar \beta$ are intrinsic time scales of the environmental fluctuations. Eq. (1) now simplifies to

$$
\begin{aligned}
\varrho_{\xi}(t)= & \mathfrak{L} \varrho_{\xi}(t):=-\frac{i}{\hbar}\left[H_{\mathrm{s}}, \varrho_{\xi}(t)\right]-\frac{i}{\hbar}\left[H_{\mathrm{c}}, \varrho_{\xi}(t)\right] \\
& -\frac{i}{2 \hbar} \gamma_{0}\left[q,\left\{p, \varrho_{\xi}(t)\right\}\right]+\frac{i}{\hbar} \xi(t)\left[q, \varrho_{\xi}(t)\right],
\end{aligned}
$$

where $\gamma_{0}$ is the damping rate of a Brownian particle of mass $m$ 18. The physical density matrix is a stochastic average of the form $\varrho(t)=\mathbb{E}\left[\varrho_{\xi}(t)\right]$.

At the price of introducing an explicit noise variable $\xi(t)$, Eq. (2) represents the exact non-Markovian dynamics in terms of a stochastic ensemble with time-local equations of motion. All memory effects inherent in the reservoir dynamics are contained in the time-dependent correlations. There is no decomposition of the environment into additional degrees of freedom and a secondary, Markovian environment [19]. In an extreme hightemperature limit, Eq. (2) becomes Markovian and reduces to the master equation of Caldeira and Leggett [20].

Optimal control means searching for control signals which drive desirable characteristics of the dynamics to extremal values. Here we consider optimization objectives defined by minimization of an expectation value $\langle M\rangle$ at a specified end time $T$. This leads to a search for extrema of the objective functional

$$
\mathrm{B}[\mathbf{u}(t)]=\mathbb{E}\left[\operatorname{tr}\left\{M \varrho_{\xi}(T)\right\}\right]=\operatorname{tr}\{M \varrho(T)\},
$$

where the first equality relies on the map $\mathbf{u} \mapsto \varrho_{\xi}(T)$ implicit in the initial-value problem of Eq. (2). Alternatively, Eq. (2) can be interpreted as a constraint on simultaneous variations of $\mathbf{u}(t)$ and $\varrho_{\xi}(t)$. This constraint needs to be taken into account through a time-dependent Lagrange multiplier $\Lambda_{\xi}(t)$, which also depends on the particular noise realization $\xi(t)$. Variational calculus leads to the equation of motion

$$
\begin{aligned}
\dot{\Lambda}_{\xi}(t)= & -\mathfrak{L}^{\dagger} \Lambda_{\xi}(t)=-\frac{i}{\hbar}\left[H_{\mathrm{s}}, \Lambda_{\xi}(t)\right]-\frac{i}{\hbar}\left[H_{\mathrm{c}}, \Lambda_{\xi}(t)\right] \\
& -\frac{i}{2 \hbar} \gamma_{0}\left\{p,\left[q, \Lambda_{\xi}(t)\right]\right\}+\frac{i}{\hbar} \xi(t)\left[q, \Lambda_{\xi}(t)\right]
\end{aligned}
$$

for $\Lambda_{\xi}(t)$, called the costate of the optimal control problem in this context. Equation (4) is not an initial value problem; it needs to be solved with the terminal boundary condition $\Lambda_{\xi}(T)+M=0$ arising from variation of the final state.

Now the gradient of the objective functional under the above constraint is given by

$$
\left.\frac{\delta \mathrm{B}}{\delta u_{j}(t)}\right|_{\text {constr. }}=\mathbb{E}\left[\operatorname{tr}\left\{\Lambda_{\xi}(t) \frac{\partial \mathfrak{L}}{\partial u_{j}} \varrho_{\xi}(t)\right\}\right],
$$

where $\varrho_{\xi}(t)$ and $\Lambda_{\xi}(t)$ obey the stochastic equations of motion (2) and (4).

The preceding considerations show that the SLN approach treats quantum memory effects in a mathematical language which integrates seamlessly into the statecostate framework of standard OCT techniques. For numerical computations, we have adapted the monotonically convergent algorithm of Krotov 21, 22] to the present case of non-Markovian stochastic propagation. This algorithm improves performance by substituting gradient search steps with a nonlocal generalization of Eq. (5). Key performance characteristics are improved by this change (see [15]).

Application.-As a common model we consider a harmonic oscillator, i.e., $H_{\mathrm{s}}=\frac{p^{2}}{2 m}+\frac{m \omega_{0}^{2}}{2} q^{2}$, which is subject to an additional potential $H_{\mathrm{c}}(t)=-F(t) q+\frac{m}{2} \Delta(t) q^{2}$ depending on a force control fields $F(t) \equiv u_{1}(t)$ and a tuning control field $\Delta(t) \equiv u_{2}(t)$, which modifies the instantaneous resonance frequency, $\omega^{2}=\omega_{0}^{2}+\Delta$. This choice is not only a model for typical realizations as, e.g., trapped atoms or ions or low energy dynamics of Josephson junctions, it also offers itself as a simple test case where an intuitive interpretation of numerical results may be feasible. It is nontrivial since it includes the nonlinear response of the system to parametric driving, and it is fairly generic as it applies to potentials with harmonic minima.

Under the equation of motion (2), the individual samples $\varrho_{\xi}$ remain Gaussian for Gaussian initial states. This allows us to rephrase the equation of motion (2) as a system of ordinary stochastic differential equations for the first and second cumulants (means and variances) associated with $\varrho_{\xi}$, i.e., $\langle q\rangle_{c},\langle p\rangle_{c},\left\langle q^{2}\right\rangle_{c},\left\langle p^{2}\right\rangle_{c}$ and $\left\langle\frac{1}{2}(q p+p q)\right\rangle_{c}$. A similar consideration holds for the costate dynamics (4) if a maximal overlap with a Gaussian target state is chosen as optimization objective, i.e., $M=1-A$, where $A=|\alpha\rangle\langle\alpha|$ projects onto a coherent state. We thus obtain closed equations of motion for the first two cumulants in the propagation of both the state $\varrho_{\xi}(t)$ and the costate $\Lambda_{\xi}(t)$. While the effect of the linear control $F(t)$ alone is given by linear response theory, the dynamical squeezing through a time-dependent $\Delta(t)$ leads to nontrivial dynamics, as does the combined action of both controls. We have explored these effects numerically, computing the expectation values in Eq. (5) explicitly through a large number of samples (typically $10^{4}$ ). This has the advantage of being securely based on first 
principles, without resorting to approximations of the dynamics.

In the following, we use natural units $\left(\hbar=k_{\mathrm{B}}=\right.$ 1, units $\omega_{0}$ for energies, angular frequencies, or rates, $1 / \sqrt{m \omega_{0}}$ for lengths, and $\sqrt{m \omega_{0}}$ for momenta). We choose a minimal-uncertainty wavepacket centered around $q=1$ and $p=0$ as both initial and target state. Values of the temperature and the damping constant are chosen in the range typical of superconducting solid-state devices [2]. The propagation time $T=20$ is roughly comparable to the relaxation time in the examples to be discussed.

We compare the results of iteratively determined control fields for three types of dynamics inserted for state and costate in Eq. (5): (a) SLN dynamics; (b) the standard Markovian master equation of the harmonic oscillator [23, with the usual raising and lowering operators associated with $H_{\mathrm{s}}$ as Lindblad operators; (c) quantum dynamics without dissipation.

Figures 1 and 2 show time-frequency signatures of the controls $F(t)$ and $\Delta(t)$ obtained through the windowed Fourier transform (also short-time Fourier transform, STFT) using a Gaussian window. Both controls show marked differences between the SLN and RWA cases. The tendency for more pronounced and more complex high-frequency features in the SLN case indicates the importance of exercising control also on time scales of the environmental fluctuations ( of order $\beta$ ), similar to a known strong-field approach to the suppression of decoherence known as 'bang-bang control' [24]. A second tendency seen in the SLN results is the application of fields spread out over the entire time interval, as compared to the emphasis on a stronger initial perturbation in the cases of RWA dissipation or no dissipation.

Values of the objective functional achieved with the SLN fields for different temperatures and damping constants are compared in Table I] Free dynamics (no control) would result in values roughly equal to $1 / 2$ for all parameters listed. A test of the control fields obtained in RWA, inserted in the exact equation of motion, typically yields values of the objective functional which are up to $100 \%$ larger than for controls computed using SLN dynamics. The algorithmic property of monotonic convergence is confirmed by our numerical results.

\begin{tabular}{c||l|l|l}
$\beta \backslash \gamma_{0}$ & 0.005 & 0.01 & 0.05 \\
\hline \hline 0.5 & 0.1036 & 0.1582 & 0.3351 \\
\hline 1.0 & 0.0477 & 0.0688 & 0.1432 \\
\hline 5.0 & 0.0059 & 0.0109 & 0.0245 \\
\hline 50.0 & 0.0037 & 0.0072 & 0.0133
\end{tabular}

Table I. Results for the minimization of $\operatorname{tr}\{M \rho(T)\}$ for various inverse temperatures $\beta$ and different damping constants $\gamma_{0}$ in the range typical of mesoscopic quantum circuits or condensed-phase chemical reactions.

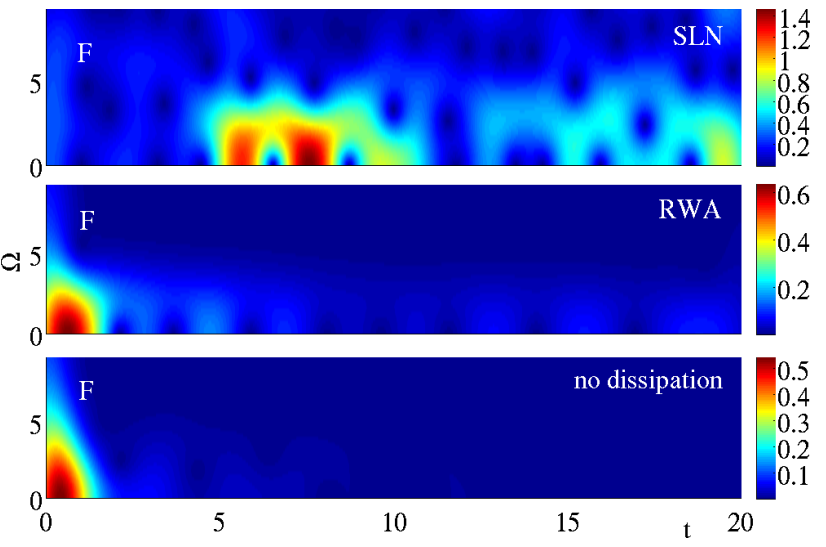

Figure 1. (color online) Windowed Fourier transform of the optimal control force $F(t)$ obtained using different dynamical equations: (a) SLN equation (2), SLN, (b) a simple generalization of the standard master equation to driven systems, RWA, and (c) unitary propagation. Parameters are $\gamma_{0}=0.05$, $\omega_{c}=50, \beta=1$.

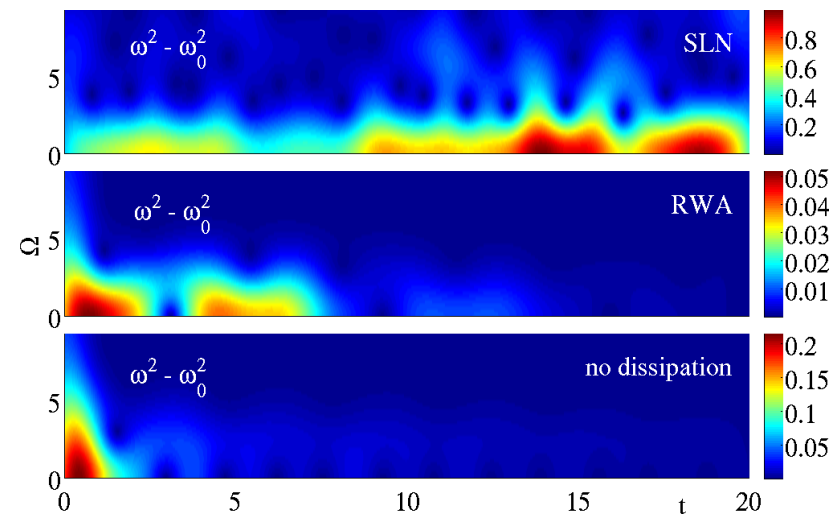

Figure 2. (color online) Windowed Fourier transform of the optimal tuning field $\Delta=\omega^{2}-\omega_{0}^{2}$ obtained using dynamical equations as in Fig. 1 Different color scales apply to the three scenarios.

Dynamical cooling.-Optimal control for closed systems conserves entropy like any unitary time evolution. Quantum dissipation invariably creates mixed states in the subsystem of interest, i.e., if the initial state is pure the entropy of the open system will increase. But can optimal control of an open system prevent this or even lower the entropy in other cases? To investigate this question, we choose the oscillator ground state as target and prepare both system and environment as thermal states with equal inverse temperature $\beta=1$. In this symmetric setting, the field $F(t)$ is not needed, since it changes the position, but not the shape of the wave packet. We therefore consider only the control field $\Delta(t)$ in the following. The von Neumann entropy of the mixed state is given by [25] $S(\varrho)=g\left(\sqrt{\left\langle q^{2}\right\rangle_{c}\left\langle p^{2}\right\rangle_{c}-\langle p q+q p\rangle_{c}^{2} / 4}\right)$ 


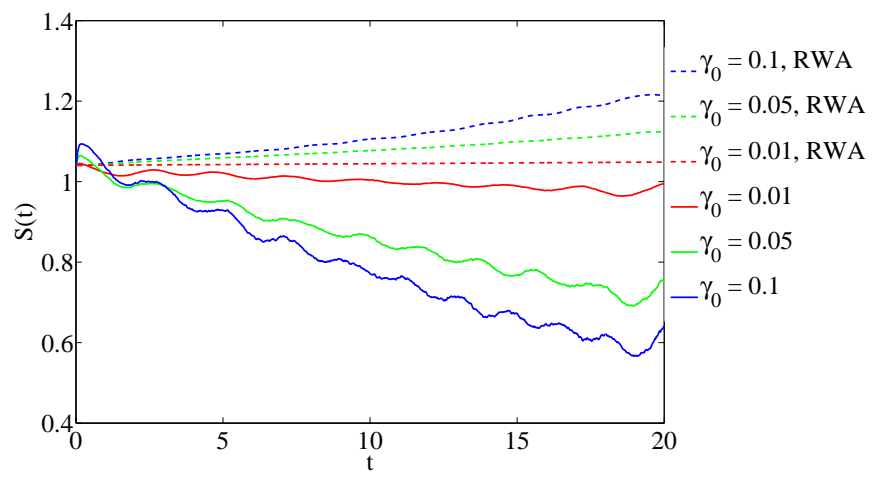

Figure 3. (color online) An open quantum system initially equilibrated with its surroundings loses entropy $S$ under an optimized control field (solid). In contrast, the standard Markovian/RWA master equation leads to increased entropy under driving (dashed, see [15]

with $g(x)=\left(x+\frac{1}{2}\right) \log \left(x+\frac{1}{2}\right)-\left(x-\frac{1}{2}\right) \log \left(x-\frac{1}{2}\right)$. We thus obtain the counterintuitive result that a timedependent control field can modify dissipative dynamics to the point where its entropy change turns negative (Fig. 3). We attribute this phenomenon to the cooperative effect of driving and dissipation, since neither of the two alone can cause this. The subsystem energy of the final state decreases below its original thermal value, indicating a dynamical cooling effect. In contrast, it can be shown (see Supplemental Material [15]) that commonly used RWA methods predict heating above the environmental temperature for non-zero driving. Consistent corrections of master equations for finite $H_{\mathrm{c}}$ prove to be a formidable challenge [11. Moreover, even if $H_{\mathrm{c}}$ could be used in the construction of the dissipator, the distinction between co- and counter-rotating terms would hardly be justified. If the control fields change on the timescale of the reservoir fluctuations, a 'wobbly frame' rather than a rotating frame results.

In contrast to recent proposals for quantum refrigerators [26, 27], which rely on intricate band or level structures, we have chosen a model with minimal structure. The cooling effect found here seems to be a feature of temporal patterns, not of a specifically designed system. We also note that no internal degree of freedom is needed for the effect to occur.

Conclusions. - The present SLN approach to optimal control enjoys two natural advantages compared to control theory based on standard Markovian master equations: (i) its noise statistics are by construction independent of the quantum dynamics, i.e., strong external driving introduces no need for correction terms, and (ii) one arrives at the usual state-costate picture required by OCT methods in a straightforward way. Numerical control of a harmonic degree of freedom is demonstrated with varying parameters and objectives. Most results show marked differences compared to the RWA approach, where the influence of driving on dissipation is neglected. Efficient computations are feasible for environmental couplings from weak damping up to a quality factor as low as $Q \approx 10$. This allows applications to solid-state devices such as superconducting circuits with Josephson junctions and condensed-matter phenomena such as reactive dynamics of small molecules in a solvent or on a surface. Optimal control of a dissipative quantum system can extract entropy from a system initially at the same temperature as its environment. Dynamical cooling in a simple system without special structural features may be considered as a likely strategy for mesoscopic quantum refrigeration.

Acknowledgements. We gratefully acknowledge helpful conversations with S. Montangero and M. Murphy as well as financial support from Land Baden-Württemberg, DFG (SFB569, SFB/TRR21), EU (Marie Curie FP7-IEF, AQUTE, DIAMANT, PICC) and Ulm University/UUG.

[1] P. Treutlein et al., Phys. Rev. Lett. 92, 203005 (2004).

[2] G. Ithier et al., Phys. Rev. B 72, 134519 (2005).

[3] S. Montangero, T. Calarco, and R. Fazio, Phys. Rev. Lett. 99, 170501 (2007).

[4] C. M. Tesch and R. de Vivie-Riedle, Phys. Rev. Lett. 89, 157901 (2002).

[5] A. Spörl et al., Phys. Rev. A 75, 012302 (2007).

[6] R. Nigmatullin and S. G. Schirmer, New J. Phys. 11, 105032 (2009).

[7] J. P. Palao and R. Kosloff, Phys. Rev. Lett. 89, 188301 (2002).

[8] T. Schulte-Herbrüggen, A. Spörl, N. Khaneja, and S. J. Glaser, Phys. Rev. A 72, 042331 (2005).

[9] P. Treutlein, T. W. Hänsch, J. Reichel, A. Negretti, M. A. Cirone, and T. Calarco, Phys. Rev. A 74, 022312 (2006).

[10] A. G. Kofman and G. Kurizki, Phys. Rev. Lett. 93, 130406 (2004).

[11] R. Xu, Y. Yan, Y. Ohtsuki, Y. Fujimura, and H. Rabitz, J. Chem. Phys. 120, 6600 (2004).

[12] J. T. Stockburger and H. Grabert, Phys. Rev. Lett. 88, 170407 (2002); J. T. Stockburger, Chem. Phys. 296, 159 (2004).

[13] W. Koch, F. Großmann, J. T. Stockburger, and J. Ankerhold, Phys. Rev. Lett. 100, 230402 (2008).

[14] See Eq. (4) and preceding paragraph.

[15] See Supplemental Material (attached) for details on our OCT approach and a comparison with standard RWA methods.

[16] U. Weiss, Quantum dissipative systems (World Scientific, Singapore, 2008), 3rd ed.

[17] Y. Tanimura, J. Phys. Soc. Jpn. 75, 082001 (2006).

[18] J. T. Stockburger and C. H. Mak, J. Chem. Phys. 110, 4983 (1999).

[19] P. Rebentrost et al., Phys. Rev. Lett. 102, 090401 (2009); T. Schulte-Herbrüggen et al., J. Phys. B 44, 154013 (2011).

[20] A. O. Caldeira and A. J. Leggett, Physica A 121, 587 (1983). 
[21] V. F. Krotov, Global methods in optimal control theory (Marcel Dekker, New York, 1996).

[22] S. E. Sklarz and D. J. Tannor, Phys. Rev. A 66, 053619 (2002).

[23] H.-P. Breuer and F. Petruccione, The theory of open quantum systems (Oxford University Press, 2002).

[24] L. Viola and S. Lloyd, Phys. Rev. A 58, 2733 (1998).

[25] A. S. Holevo, M. Sohma, and O. Hirota, Phys. Rev. A 59, 1820 (1999).

[26] J. P. Pekola and F. W. J. Hekking, Phys. Rev. Lett. 98, 210604 (2007).

[27] M. Rey, M. Strass, S. Kohler, P. Hänggi, and F. Sols, Phys. Rev. B 76, 085337 (2007). 


\title{
EPAPS for "Optimal control of open quantum systems: cooperative effects of driving and dissipation"
}

\author{
R. Schmidt, ${ }^{1}$ A. Negretti, ${ }^{2}$ J. Ankerhold, ${ }^{1}$ T. Calarco, ${ }^{2}$ and J. T. Stockburger ${ }^{1}$ \\ ${ }^{1}$ Institut für Theoretische Physik, Universität Ulm, Albert-Einstein-Allee 11, 89069 Ulm, Germany. \\ ${ }^{2}$ Institut für Quanteninformationsverarbeitung, Universität \\ Ulm, Albert-Einstein-Allee 11, 89069 Ulm, Germany.
}

\begin{abstract}
This document serves as a supplement to our article "Optimal control of open quantum systems: cooperative effects of driving and dissipation" [Physical Review Letters (2011)]. It describes the numerical algorithm used, a generalization of the Krotov iterative algorithm to open quantum systems evolving under a stochastic equation of motion. A comparison with the Markovian dynamics of a commonly used quantum master equation shows that the latter cannot reproduce the cooperative cooling effect described in the main text.
\end{abstract}

\section{OBJECTIVE AND CONSTRAINT}

We outline a modification of Krotov's algorithm for the optimization of final-state properties of an open quantum system governed by the stochastic differential equation

$$
\dot{\hat{\varrho}}_{\xi}(t)=\hat{\mathfrak{L}} \hat{\varrho}_{\xi}(t):=-\frac{i}{\hbar}\left[\hat{H}_{\mathrm{s}}, \hat{\varrho}_{\xi}(t)\right]-\frac{i}{\hbar}\left[\hat{H}_{\mathrm{c}}(t), \hat{\varrho}_{\xi}(t)\right]-\frac{i}{2 \hbar} \gamma_{0}\left[\hat{q},\left\{\hat{p}, \hat{\varrho}_{\xi}(t)\right\}\right]+\frac{i}{\hbar} \xi(t)\left[\hat{q}, \hat{\varrho}_{\xi}(t)\right],
$$

which must be considered as a dynamical constraint in the context of the optimization problem. Here $\gamma_{0}$ is the damping rate of a Brownian particle in the (ohmic) environment, and $\xi(t)$ is a noise variable whose statistics are governed by the quantum correlation function of the environmental fluctuations.

The objective functionals to be minimized is assumed to depend linearly on the terminal state, i.e., the functional is the expectation value of an observable $\hat{M}$ at the final time $T$, typically a projection operator. The physical terminal state $\hat{\varrho}(T)$ is the stochastic average of the dynamical state $\hat{\varrho}_{\xi}(T)$, i. e., the objective functional is of the form

$$
\mathrm{B}[\mathbf{u}(t)]=\operatorname{tr}\{\hat{M} \hat{\varrho}(T)\}=\mathbb{E}\left[\operatorname{tr}\left\{\hat{M} \hat{\varrho}_{\xi}(T)\right\}\right]
$$

where the vector $\mathbf{u}(t)$ denotes control fields which define the time-dependence of $\hat{H}_{\mathrm{c}}(t)$, and the first equality implies the equation of motion (1). We use the symbol $\mathbb{E}$ for the expectation value with respect to the noise $\xi(t)$ only, not for the further average given by tracing over $\varrho_{\xi}(t)$. 


\section{EXTENDED OBJECTIVE FUNCTIONAL AND OPTIMAL CONTROL EQUATIONS}

Following Refs. [20, 21] (see main text), we use an extended objective functional

$$
\mathrm{L}_{\xi}\left[\mathbf{u}(t), \hat{\varrho}_{\xi}(t) ; \phi_{\xi}\right]:=\mathrm{G}_{\xi}\left(\hat{\varrho}_{\xi}(T)\right)-\int_{0}^{T} \mathrm{~d} t \mathrm{R}_{\xi}\left(t, \mathbf{u}(t), \hat{\varrho}_{\xi}(t)\right)-\phi_{\xi}\left(0, \hat{\varrho}_{\xi}(0)\right)
$$

containing the auxiliary, real-valued function $\phi_{\xi}\left(t, \varrho_{\xi}(t)\right)$, which depends on the time $t$, the noise realization $\xi(t)$, and the state sample $\hat{\varrho}_{\xi}(t)$. Further auxiliary functions

$$
\begin{aligned}
\mathrm{G}_{\xi}\left(\hat{\varrho}_{\xi}(T)\right) & :=\operatorname{tr}\left\{\hat{M} \hat{\varrho}_{\xi}(T)\right\}+\phi_{\xi}\left(T, \varrho_{\xi}(T)\right), \\
\mathrm{K}_{\xi}\left(t, \mathbf{u}(t), \hat{\varrho}_{\xi}(t), \cdot\right) & :=\operatorname{tr}\left\{\cdot \hat{\mathfrak{L}} \hat{\varrho}_{\xi}(t)\right\} \\
\mathrm{R}_{\xi}\left(t, \mathbf{u}(t), \hat{\varrho}_{\xi}(t)\right) & :=\mathrm{K}_{\xi}\left(t, \mathbf{u}(t), \hat{\varrho}_{\xi}(t), \frac{\partial}{\partial \hat{\varrho}_{\xi}} \phi_{\xi}\left(t, \varrho_{\xi}(t)\right)\right)+\frac{\partial}{\partial t} \phi_{\xi}\left(t, \hat{\varrho}_{\xi}(t)\right)
\end{aligned}
$$

are chosen such that the expectation value $\mathbb{E}\left[\mathrm{L}_{\xi}\right]$ of the extended functional coincides with the ordinary objective functional for admissible processes, i. e., pairs $\left(\mathbf{u}(t),\left\{\hat{\varrho}_{\xi}(t)\right\}\right)$ of a vector of control fields and a stochastic ensemble $\left\{\hat{\varrho}_{\xi}(t)\right\}$ with the property that the equation of motion (1) is obeyed for the given vector $\mathbf{u}(t)$ and for all noise realizations of $\xi(t)$. In this case the partial derivatives in the definition of $\mathrm{R}_{\xi}$ combine to form a total derivative, effectively canceling the boundary terms of $\phi_{\xi}$, contained in Eqs. (3) and (4).

Different choices of $\phi_{\xi}$ modify the objective functional for non-admissible processes, such as the combination of control fields and states from different iterations. For Krotov's algorithm it is necessary to choose a function $\phi_{\xi}$ with the following property: For any admissible process, $L_{\xi}$ is at a maximum with respect to variations of $\hat{\varrho}_{\xi}$ with the function $\mathbf{u}(t)$ kept fixed, i. e., variations away from the submanifold of admissible processes decrease $L_{\xi}$. This means that an appropriate choice of $\phi_{\xi}$ must conform to the two conditions

$$
\begin{aligned}
\mathrm{G}_{\xi}\left(\hat{\varrho}_{\xi}^{(j)}(T)\right) & =\max _{\hat{\varrho}_{\xi}}\left\{\mathrm{G}_{\xi}\left(\hat{\varrho}_{\xi}(T)\right)\right\}, \\
\mathrm{R}_{\xi}\left(t, \mathbf{u}^{(j)}(t), \hat{\varrho}_{\xi}^{(j)}(t)\right) & =\min _{\hat{\varrho}_{\xi}}\left\{\mathrm{R}_{\xi}\left(t, \mathbf{u}^{(j)}(t), \hat{\varrho}_{\xi}(t)\right)\right\},
\end{aligned}
$$

where the superscript $(j)$ denotes the admissible process resulting from an initial guess or a previous iteration.

After making such a choice of $\phi_{\xi}^{(j)}$, we demand that, in addition to being admissible, the new process $\left(\mathbf{u}^{(j+1)}(t),\left\{\hat{\varrho}_{\xi}^{(j+1)}(t)\right\}\right)$ is taken from the class of pairs $\left(\tilde{\mathbf{u}}(t),\left\{\tilde{\varrho}_{\xi}(t)\right\}\right)$ which fulfill the condition

$$
\tilde{\mathbf{u}}(t)=\arg \max _{\mathbf{u}(t)} \mathbb{E}\left[\mathrm{R}_{\xi}\left(t, \mathbf{u}(t), \tilde{\varrho}_{\xi}(t)\right)\right]
$$


at any time $t$. This condition selects a specific pair $\left(\mathbf{u}^{(j+1)}(t),\left\{\hat{\varrho}_{\xi}^{(j+1)}(t)\right\}\right)$ from the set of admissible process. Using Eq. (9), the update $\mathbf{u}^{(j)}(t) \rightarrow \mathbf{u}^{(j+1)}(t)$ can be performed locally in conjunction with the propagation of the new state $\hat{\varrho}_{\xi}^{(j+1)}(t)$ since the dependence of the dynamical state on the control fields obeys causality.

What remains to be specified is a mathematical construction which defines specific computational steps using the function $\phi_{\xi}$. For the linear dynamics of Eq. (1), it turns out that the function $\phi_{\xi}\left(t, \hat{\varrho}_{\xi}\right)$ appears in the conditions (7) and (8) only in form of its derivative with respect to the state, and since a complete derivative can be formed from them, the co-state $\hat{\Lambda}_{\xi}=\partial \phi_{\xi} / \partial \hat{\varrho}_{\xi}$ can be characterized as an additional dynamical variable with the equation of motion

$$
\dot{\hat{\Lambda}}_{\xi}(t)=-\hat{\mathfrak{L}}^{\dagger} \hat{\Lambda}_{\xi}(t)=-\frac{i}{\hbar}\left[\hat{H}_{\mathrm{s}}, \hat{\Lambda}_{\xi}(t)\right]-\frac{i}{\hbar}\left[\hat{H}_{\mathrm{c}}(t), \hat{\Lambda}_{\xi}(t)\right]-\frac{i}{2 \hbar} \gamma_{0}\left\{\hat{p},\left[\hat{q}, \hat{\Lambda}_{\xi}(t)\right]\right\}+\frac{i}{\hbar} \xi(t)\left[\hat{q}, \hat{\Lambda}_{\xi}(t)\right]
$$

Solving this equation with the end-time boundary condition $\hat{\Lambda}_{\xi}(T)+\hat{M}=0$, resulting from (7), amounts to a (fully sufficient) construction of $\phi_{\xi}$ to first order in variations of $\hat{\varrho}_{\xi}$ around $\hat{\varrho}_{\xi}^{(j)}$.

In a variant of the algorithm, described by Sklarz and Tannor [21], the implicit condition (9) is substituted by an explicit form

$$
u_{k}^{(j+1)}(t)=u_{k}^{(j)}(t)+\frac{1}{\lambda_{k}(t)} \mathbb{E}\left[\operatorname{tr}\left\{\hat{\Lambda}_{\xi}^{(j)}(t) \frac{\partial \hat{\mathfrak{L}}}{\partial u_{k}} \hat{\varrho}_{\xi}^{(j+1)}(t)\right\}\right]
$$

which is equivalent to Eq. (9) if a suitable ( $j$-dependent) cost functional is added to B. It is to be noted that the simultaneous appearance of $j$ and $j+1$ on the r.h.s. of this equation gives this update law properties quite different from a simple gradient search. The functions $\lambda_{k}(t)$ arising from this cost functional may be adjusted to tune convergence properties. In summary, the algorithm works as follows:

1. An initial guess for the control fields $\mathbf{u}^{(0)}(t)$ is chosen.

2. The equation of motion $(1)$ with $\mathbf{u}^{(0)}(t)$ is solved with initial condition $\hat{\varrho}(0)$ for an ensemble of trajectories $\left\{\hat{\varrho}_{\xi}(t)\right\}$.

3. The co-state ensemble is initialized using the condition $\hat{\Lambda}_{\xi}(T)+\hat{M}=0$ and then propagated backwards to the initial time with Eq. (10).

4. The ensembles $\left\{\hat{\varrho}_{\xi}(t)\right\}$ and $\left\{\hat{\Lambda}_{\xi}(t)\right\}$ are propagated forward until the end time $T$, but at each time step the co-states are propagated with the old control fields, that is, the ones obtained from the $j$-th iteration, whereas the states $\hat{\varrho}_{\xi}(t)$ are propagated with the new control fields given by the update law (11). (Alternatively, stored values from step 3 can be used). 
5. The objective functional (2) is computed, and steps 3 and 4 are repeated until the value of objective functional is in the desired range.

This optimization algorithm applies not only to Eq. (1), but to any type of linear dynamics subject to additive or multiplicative noise.

\section{PROOF OF MONOTONIC CONVERGENCE}

The procedure outlined above guarantees that each step of the iteration reduces the objective functional, that is, $\mathbb{E}\left[\mathrm{B}_{\xi}\left[\mathbf{u}^{(j+1)}(t), \hat{\varrho}_{\xi}^{(j+1)}(t)\right]\right] \leq \mathbb{E}\left[\mathrm{B}_{\xi}\left[\mathbf{u}^{(j)}(t), \hat{\varrho}_{\xi}^{(j)}(t)\right]\right]$. This becomes evident if the change of the objective functional is decomposed into three terms,

$$
\mathbb{E}\left[\mathrm{L}_{\xi}\left[\mathbf{u}^{(j)}(t), \hat{\varrho}_{\xi}^{(j)}(t), \phi_{\xi}\right]\right]-\mathbb{E}\left[\mathrm{L}_{\xi}\left[\mathbf{u}^{(j+1)}(t), \hat{\varrho}_{\xi}^{(j+1)}(t), \phi_{\xi}\right]\right]=\Delta_{1}+\Delta_{2}+\Delta_{3},
$$

where

$$
\begin{aligned}
& \Delta_{1}=\int_{0}^{T} \mathrm{~d} t \mathbb{E}\left[\mathrm{R}\left(t, \mathbf{u}^{(j)}(t), \hat{\varrho}_{\xi}^{(j+1)}(t)\right)-\mathrm{R}\left(t, \mathbf{u}^{(j)}(t), \hat{\varrho}_{\xi}^{(j)}(t)\right)\right] \\
& \Delta_{2}=\int_{0}^{T} \mathrm{~d} t \mathbb{E}\left[\mathrm{R}\left(t, \mathbf{u}^{(j+1)}(t), \hat{\varrho}_{\xi}^{(j+1)}(t)\right)-\mathrm{R}\left(t, \mathbf{u}^{(j)}(t), \hat{\varrho}_{\xi}^{(j+1)}(t)\right)\right] \\
& \Delta_{3}=\mathbb{E}\left[\mathrm{G}\left(\hat{\varrho}_{\xi}^{(j)}(T)\right)-\mathrm{G}\left(\hat{\varrho}_{\xi}^{(j+1)}(T)\right)\right]
\end{aligned}
$$

Now $\Delta_{1} \geq 0$ and $\Delta_{3} \geq 0$ follow from Eq. (8) and Eq. (7), respectively, whereas $\Delta_{2} \geq 0$ is a property of the update law (9). We have thus demonstrated monotonicity for the modified algorithm, which ensures convergence for an arbitrary initial guess with any objective functional bounded from below.

Recent work by S. Schirmer and P. de Fouquieres [New J. Phys. 13, 073029 (2011)] points out that proofs of monotonicity for Krotov-type iteration methods are not rigorous if time-discrete approximations are substituted for the dynamics of state and co-state. For all practical purposes, however, the typical benefits of the Krotov method persist, i. e., convergence for a wide range of initial guesses and rapid improvement during the first iteration steps.

\section{DRIVEN DYNAMICS WITH FIXED RWA DISSIPATOR: EFFECTIVE RESERVOIR}

For comparison, we study the control problem of the parametrically driven harmonic oscillator, modeling the open system dynamics through a Lindblad Master equation. From a system-reservoir model, this equation results from performing the Born, Markov, and RWA approximations on the 
system-reservoir interactions. In the dimensionless units introduced in the main text, the Master equation reads

$$
\begin{aligned}
\frac{d}{d t} \hat{\rho} & =-i[\hat{H}(t), \hat{\rho}] \\
& +\gamma_{0}\left(N_{\beta}+1\right)\left(\hat{a} \hat{\rho} \hat{a}^{\dagger}-\frac{1}{2}\left\{\hat{a}^{\dagger} \hat{a}, \hat{\rho}\right\}\right) \\
& +\gamma_{0} N_{\beta}\left(\hat{a}^{\dagger} \hat{\rho} \hat{a}-\frac{1}{2}\left\{\hat{a} \hat{a}^{\dagger}, \hat{\rho}\right\}\right)
\end{aligned}
$$

with

$$
\hat{H}(t)=\hat{a}^{\dagger} \hat{a}+\frac{1}{2}+\frac{\Delta(t)}{4}\left(\hat{a}+\hat{a}^{\dagger}\right)^{2}=\left(1+\frac{\Delta(t)}{2}\right)\left(\hat{a}^{\dagger} \hat{a}+\frac{1}{2}\right)+\frac{\Delta(t)}{4}\left(\hat{a}^{2}+\hat{a}^{\dagger 2}\right)
$$

Following common practice (see, however, Refs. $[10,11]$ ), we have defined the rotating frame of the RWA through the time-independent Hamiltonian $\hat{H}_{0}=\hat{a}^{\dagger} \hat{a}+\frac{1}{2}$, not the full Hamiltonian $\hat{H}(t)$. Accordingly, the dissipative terms of Eq. (16) contain the thermal occupation number $N_{\beta}=\left(e^{\beta}-1\right)^{-1}$ of the unperturbed oscillator.

Due to the mismatch between the Hamiltonians of the pre-set rotating frame and of the driven dynamics, oscillatory terms reappear when the dissipative terms are transformed to the interaction picture. In order to show this, we define the interaction picture with respect to $\hat{H}(t)$,

$$
\hat{\rho}(t)=\hat{U}(t) \hat{\rho}_{\mathrm{I}}(t) \hat{U}^{\dagger}(t), \quad \dot{\hat{U}}=i \hat{H} \hat{U}
$$

The Master equation for $\hat{\rho}_{\mathrm{I}}$ then reads

$$
\begin{aligned}
\frac{d}{d t} \hat{\rho}_{\mathrm{I}} & =\gamma_{0}\left(N_{\beta}+1\right)\left(\hat{b} \hat{\rho}_{\mathrm{I}} \hat{b}^{\dagger}-\frac{1}{2}\left\{\hat{b}^{\dagger} \hat{b}, \hat{\rho}_{\mathrm{I}}\right\}\right) \\
& +\gamma_{0} N_{\beta}\left(\hat{b}^{\dagger} \hat{\rho}_{\mathrm{I}} \hat{b}-\frac{1}{2}\left\{\hat{b} \hat{b}^{\dagger}, \hat{\rho}_{\mathrm{I}}\right\}\right)
\end{aligned}
$$

where $\hat{b}=\hat{U}^{\dagger} \hat{a} \hat{U}$ and its adjoint are time dependent, following the equations of motion

$$
\begin{aligned}
\dot{\hat{b}} & =-i\left(1+\frac{\Delta}{2}\right) \hat{b}-i \frac{\Delta}{2} \hat{b}^{\dagger} \\
\dot{\hat{b}}^{\dagger} & =i \frac{\Delta}{2} \hat{b}+i\left(1+\frac{\Delta}{2}\right) \hat{b}^{\dagger} .
\end{aligned}
$$

From this, one easily concludes that $b$ is a linear combination of $\hat{a}$ and $\hat{a}^{\dagger}$ with $\left[\hat{b}, \hat{b}^{\dagger}\right]=1$, i.e., the map $\hat{a} \mapsto \hat{b}$ is a Bogoliubov transformation

$$
\hat{b}=e^{i \theta} \cosh y \hat{a}+e^{i \varphi} \sinh y \hat{a}^{\dagger}
$$

with real (and, in our case, time-dependent) parameters $\theta, \phi$, and $y$. Using this equation, we obtain 
the form

$$
\begin{aligned}
\frac{d}{d t} \hat{\rho}_{\mathrm{I}} & =\gamma_{0}\left(N_{\beta}+1\right) \cosh ^{2} y\left(\hat{a} \hat{\rho}_{\mathrm{I}} \hat{a}^{\dagger}-\frac{1}{2}\left\{\hat{a}^{\dagger} \hat{a}, \hat{\rho}_{\mathrm{I}}\right\}\right) \\
& +\gamma_{0}\left(N_{\beta}+1\right) \sinh ^{2} y\left(\hat{a}^{\dagger} \hat{\rho}_{\mathrm{I}} \hat{a}-\frac{1}{2}\left\{\hat{a} \hat{a}^{\dagger}, \hat{\rho}_{\mathrm{I}}\right\}\right) \\
& +\gamma_{0} N_{\beta} \cosh ^{2} y\left(\hat{a}^{\dagger} \hat{\rho_{\mathrm{I}}} \hat{a}-\frac{1}{2}\left\{\hat{a} \hat{a}^{\dagger}, \hat{\rho}_{\mathrm{I}}\right\}\right) \\
& +\gamma_{0} N_{\beta} \sinh ^{2} y\left(\hat{a} \hat{\rho}_{\mathrm{I}} \hat{a}^{\dagger}-\frac{1}{2}\left\{\hat{a}^{\dagger} \hat{a}, \hat{\rho}_{\mathrm{I}}\right\}\right) \\
& +\gamma_{0}\left(2 N_{\beta}+1\right) \sinh y \cosh y e^{i(\theta-\varphi)}\left(\hat{a} \hat{\rho}_{\mathrm{I}} \hat{a}-\frac{1}{2}\left\{\hat{a}^{2}, \hat{\rho}_{\mathrm{I}}\right\}\right) \\
& +\gamma_{0}\left(2 N_{\beta}+1\right) \sinh y \cosh y e^{-i(\theta-\varphi)}\left(\hat{a}^{\dagger} \hat{\rho}_{\mathrm{I}} \hat{a}^{\dagger}-\frac{1}{2}\left\{\hat{a}^{\dagger}, \hat{\rho}_{\mathrm{I}}\right\}\right)
\end{aligned}
$$

for the Master equation, where all of the prefactors depend on time through $\theta, \varphi$, and $y$. Typical solutions lead to oscillating prefactors, meaning that Eq. (23) can be further simplified through time coarse graining. This in itself can be seen as evidence that the initial application of the RWA was not consistent. However, coarse-graining at this point is appropriate if one wants to compare the features of the dynamics predicted by Eqs. (16) and (23) with the exact dynamics. The first four terms of Eq. (23) contain functions of time which are squares of real time-dependent functions, therefore they typically dominate the result of the coarse graining procedure. Collecting the dominant terms results in the simplified Master equation

$$
\begin{aligned}
\frac{d}{d t} \hat{\rho}_{\mathrm{I}} & =\gamma_{0}(\tilde{N}+1)\left(\hat{a} \hat{\rho}_{\mathrm{I}} \hat{a}^{\dagger}-\frac{1}{2}\left\{\hat{a}^{\dagger} \hat{a}, \hat{\rho}_{\mathrm{I}}\right\}\right) \\
& +\gamma_{0} \tilde{N}\left(\hat{a}^{\dagger} \hat{\rho}_{\mathrm{I}} \hat{a}-\frac{1}{2}\left\{\hat{a} \hat{a}^{\dagger}, \hat{\rho}_{\mathrm{I}}\right\}\right)
\end{aligned}
$$

with

$$
2 \tilde{N}+1=\left(2 N_{\beta}+1\right) \overline{\cosh (2 y)} \geq 2 N_{\beta}+1,
$$

where the bar indicates time coarse graining. The immediate interpretation of this result is obvious: Up to a unitary transform, the dynamics of Eqs. (16) and (23) effectively describes relaxation towards a thermal oscillator state with a higher-than-thermal occupation number $\tilde{N}$. This state therefore has a higher entropy than the thermal state obtained by equilibrating without driving. Accordingly, with the dynamics approximated by Eq. (16), optimal control cannot find solutions which push the system entropy below its equilibrium value. This is consistent with our numerical findings, in particular the observation that optimal control based on Eq. (16), with overlap to the ground state as objective, converges to the trivial result $\Delta \equiv 0$. The alluringly simple method of constructing the dissipator based on a Hamiltonian without driving is an oversimplification which fails to reproduce cooperative effects of driving and dissipation, most notably, the cooling effect reported in the main text. 